\title{
Development of 6 DOF Supernumerary Robotic Fingers Integrated with 3D Animation
}

\author{
Mochammad Ariyanto,", Joga D. Setiawan, Munadi, Rifky Ismail, and Zainal Arifin \\ Department of Mechanical Engineering, Diponegoro University, Semarang, Indonesia
}

\begin{abstract}
The paper presents the development of wearable robot called supernumerary robotic fingers (SRF) that can be integrated with 3D Animation. The robot comprises of two parts, robotic finger (RF) and robotic thumb (RT). RF is located next to little finger, and RT is located next to the thumb. The robot is driven by modified glove sensor. 3D CAD model is exported into SimMechanics for 3D Animation purpose under MATLAB/Simulink environment. Forward kinematics of the robot is studied using SimMechanics and the trajectory of each robotic fingertip will be calculated. Based on the experimental result, the robot and its $3 \mathrm{D}$ animation can be operated by a healthy person in real time. The robot can assist in object manipulation with ten-inch tablet using single human hand.
\end{abstract}

\section{Introduction}

In wearable robot, a supernumerary robot is an additional robotic limb that is used to help users in holding, lifting, manipulating objects, and making it easier to do a job. Supernumerary robotic limbs (SRLs) can be fingers, arms, or legs. Supernumerary robotic fingers (SRF) has been extensively researched by the team from MIT [1-4]. The glove worn by user comprises of three stretch sensor. Based on the research works, the SRF and the human finger can work together to perform object manipulation tasks with single hand. SRF has been developed as an alternative device for prostheses and exoskeletons.

In this research, we develop a six degree of freedom (DOF) SRF as can be shown in figure 1. The robot comprises of two parts, robotic finger (RF) and robotic thumb (RT). RF is located next to little finger and RT is located next to the thumb as shown in figure 1 (b). The robot is designed by using SolidWorks CAD software. Both of the RF and the RT use six servos as the actuator and have 3 DOF. RT moves like a thumb in human hand having motion for circumduction, abduction, and flexion, while the RF moves like one of the human hand fingers having motion for abduction, and two flexion. The SRF will be attached on the wearer wrist/user. The human hand will wear modified glove sensor comprising three bend/flex sensor. The flex sensor has been utilized in previous research and can be used as input signal for robotic hand [5]. The sensor will read the bending motion of thumb, index, and middle finger of the human hand. The signal from sensor will be processed by microcontroller. It will drive the SRF and 3D animation simultaneously.

\footnotetext{
*Corresponding author: ari_janto5@yahoo.co.id
} 


\section{Design and Prototype}

The posture of SR fingers is driven by modified glove sensor. It uses three flex sensors that are attached on thumb, index, and middle on the finger gloves. The flex sensors read the bending of the user's fingers and send it to the microcontroller. The virtual robot of SR fingers is developed in SimMechanics exported from CAD model in SolidWorks as shown in figure 1 (a). The weight of the final SRF is about 650 grams excluding battery. The battery is placed on the body of user to reduce the weight of SRF. Metal servo bracket is utilized to join between two medium servos.

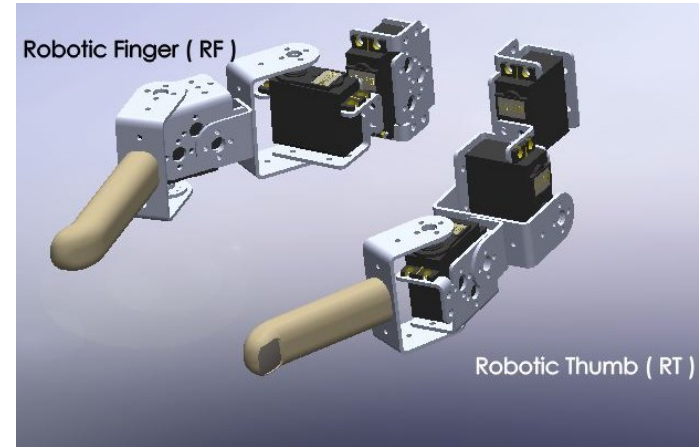

(a)

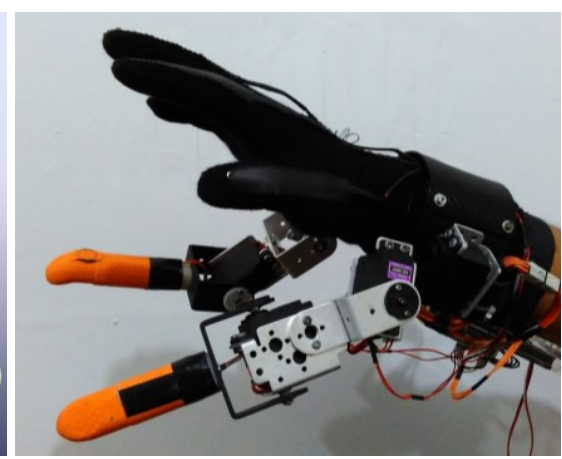

(b)

Fig. 1. SRF design (a) CAD model, (b) Prototype.

\section{Kinematics}

For forward kinematics of RT and RF, Denavit-Hartenberg (DH) parameter is utilized to study the forward kinematics from each joint input to the fingertip position of RT and RF. Each of RT and RF on SRF has three link and three revolute joints. The D-H parameter of RT and RF can be summarized in Table 1 and Table 2 respectively.

Table 1. D-H parameter of robotic thumb

\begin{tabular}{|c|c|c|c|c|}
\hline Link & $a_{i}(\mathbf{m m})$ & $a_{i}$ (degree) & $d_{i}(\mathbf{m m})$ & $\boldsymbol{\theta}_{\boldsymbol{i}}$ (degree) \\
\hline 1 & 25 & $90^{\circ}$ & 0 & $\boldsymbol{\theta}_{\boldsymbol{1}}$ \\
\hline 2 & 75 & $90^{\circ}$ & 0 & $\boldsymbol{\theta}_{2}$ \\
\hline 3 & 116 & $0^{\circ}$ & 0 & $\boldsymbol{\theta}_{3}$ \\
\hline
\end{tabular}

Table 2. D-H parameter of robotic finger

\begin{tabular}{|l|l|l|l|l|}
\hline Link & $\boldsymbol{a}_{\boldsymbol{i}}(\mathbf{m m})$ & $\boldsymbol{a}_{\boldsymbol{i}}$ (degree) & $\boldsymbol{d}_{\boldsymbol{i}}(\mathbf{m m})$ & $\boldsymbol{\theta}_{\boldsymbol{i}}$ (degree) \\
\hline 4 & 25 & $90^{\circ}$ & 0 & $\boldsymbol{\theta}_{1}$ \\
\hline 5 & 75 & $0^{\circ}$ & 0 & $\boldsymbol{\theta}_{2}$ \\
\hline 6 & 116 & $0^{\circ}$ & 0 & $\boldsymbol{\theta}_{3}$ \\
\hline
\end{tabular}


Based on the D-H parameter summarized in Table 1 and Table 2, the position of end effector in RT can be expressed from equation (1) to (3), and the end effector position of $\mathrm{RF}$ can be written from equation (4) to (6) as follows

$$
\begin{gathered}
X=a_{1} \cos \theta_{1}+a_{2} \cos \theta_{1} \cos \theta_{2}+a_{3} \sin \theta_{1} \sin \theta_{3}+a_{3} \cos \theta_{1} \cos \theta_{2} \cos \theta_{3} \\
Y=a_{1} \sin \theta_{1}+a_{2} \sin \theta_{1} \cos \theta_{2}-a_{3} \cos \theta_{1} \sin \theta_{3}+a_{3} \sin \theta_{1} \cos \theta_{2} \cos \theta_{3} \\
Z=a_{2} \sin \theta_{2}+a_{3} \sin \theta_{2} \cos \theta_{3} \\
X=a_{4} \cos \theta_{4}+a_{5} \cos \theta_{4} \cos \theta_{5}+a_{6} \cos \theta_{4} \cos \theta_{5} \cos \theta_{6}-a_{6} \cos \theta_{4} \sin \theta_{5} \sin \theta_{6} \\
Y=a_{4} \sin \theta_{4}+a_{5} \sin \theta_{4} \cos \theta_{5}+a_{6} \sin \theta_{4} \cos \theta_{5} \cos \theta_{6}-a_{6} \sin \theta_{4} \sin \theta_{5} \sin \theta_{6} \\
Z=a_{5} \sin \theta_{6}+a_{6} \cos \theta_{5} \sin \theta_{6}+a_{6} \sin \theta_{5} \cos \theta_{6}
\end{gathered}
$$

In this section, each joint angles in both RT and RF will be given with predefined angles as shown in figure 2 and figure 3 . The trajectory of the end effector in both RT and RF can be calculated using equation from (1) to equation (6), or it can be computed by using SimMechanics Software. The resulted trajectory of the end effector can be seen in Fig. 4 (a) for robotic thumb tip and figure 4 (b) for robotic fingertip. Based on the simulation result, RT tip and RF tip do not contact to each other.
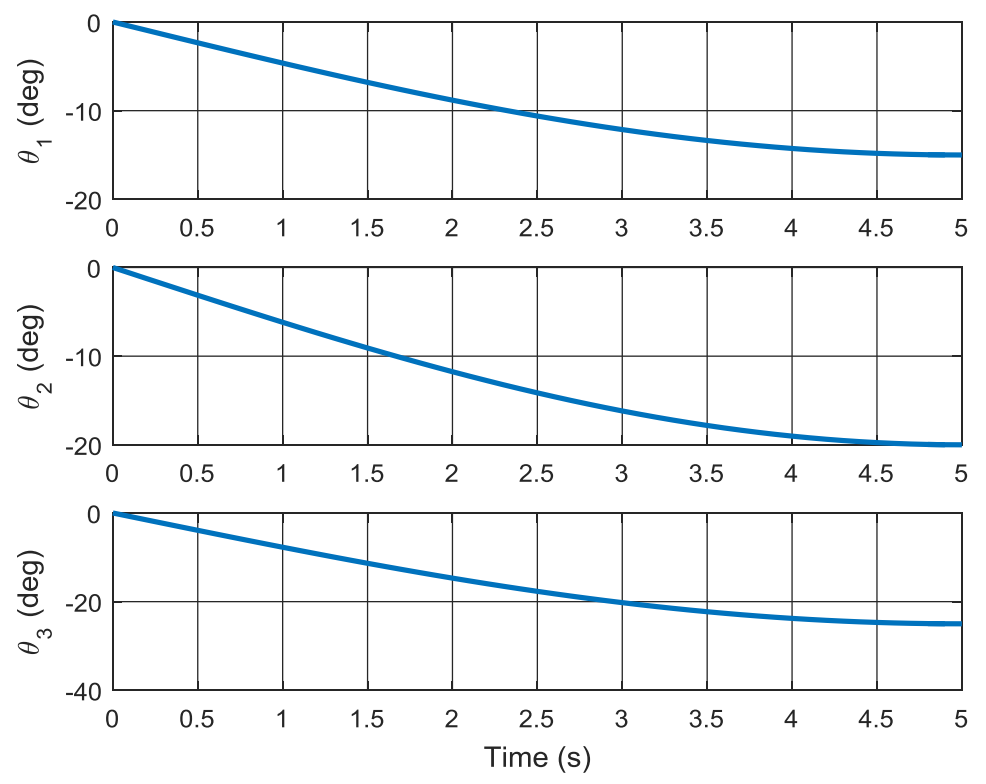

Fig. 2. Robotic thumb angle inputs. 

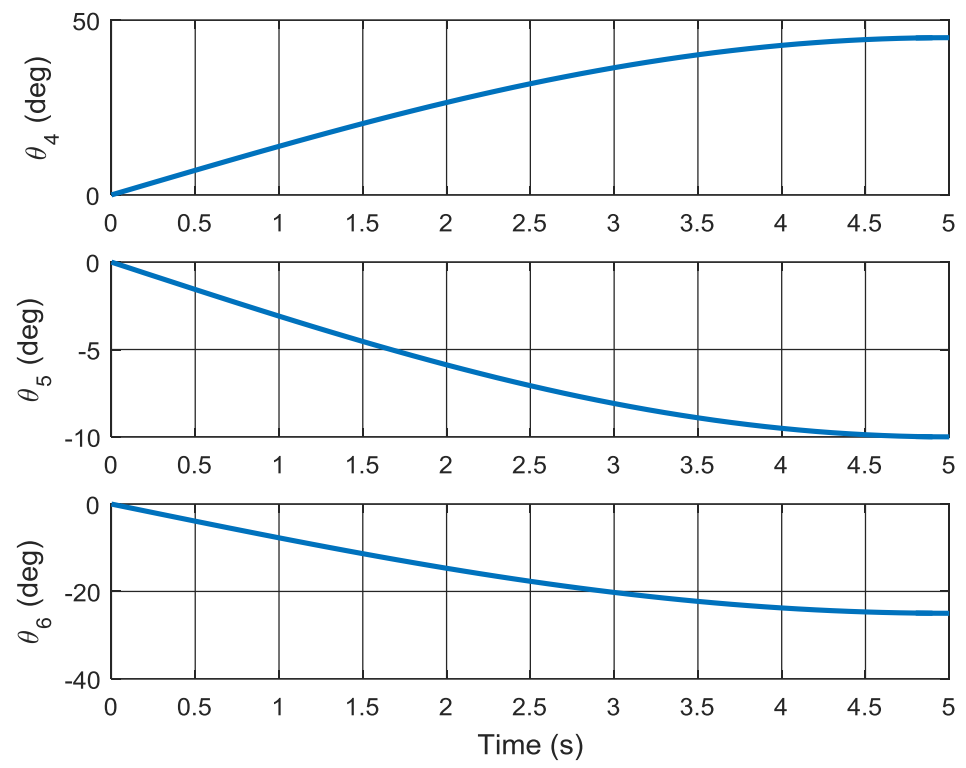

Fig. 3. Robotic finger angle inputs.

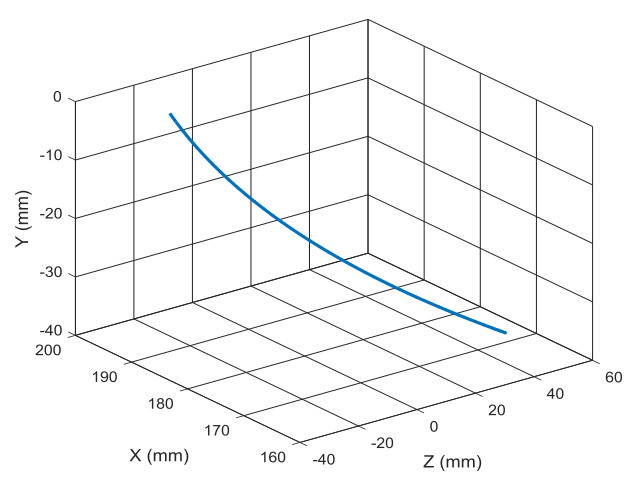

(a)

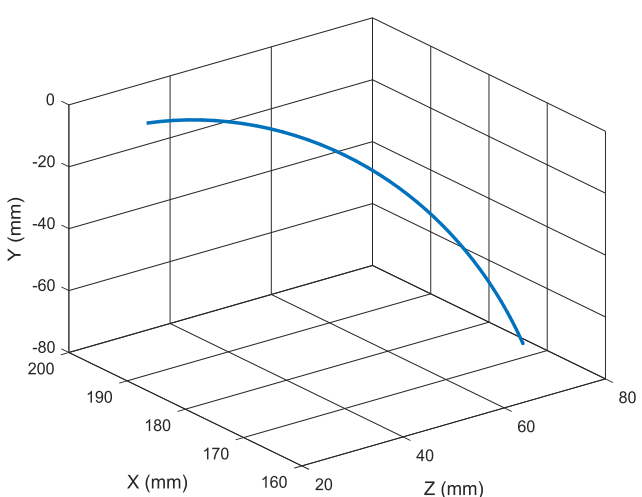

(b)

Fig. 4. Trajectory of robotic fingertips, (a) robotic thumb tip, (b) robotic fingertip

\section{SRF with 3D Animation}

For visualizing the motion both RF and RT, we develop 3D Animation of SRF that is built in SimMechanics environment. For fast transmission data between SRF and host computer, the selected baud rate is $115.200 \mathrm{bit} / \mathrm{s}$. Based on the previous research, the virtual hand or $3 \mathrm{D}$ animation of robotic hand can run simultaneously with robotic hand [6,7]. The 3D CAD model of SRF as shown in figure 1 (a) is exported from SolidWorks software to SimMechanics First Generation. The resulted of SRF block diagram is presented in figure 5. This model is run in a host computer. The test of SRF integrated with $3 \mathrm{D}$ animation is presented in figure 6. Based on the test result, SRF and 3D animation can work simultaneously in real time. 


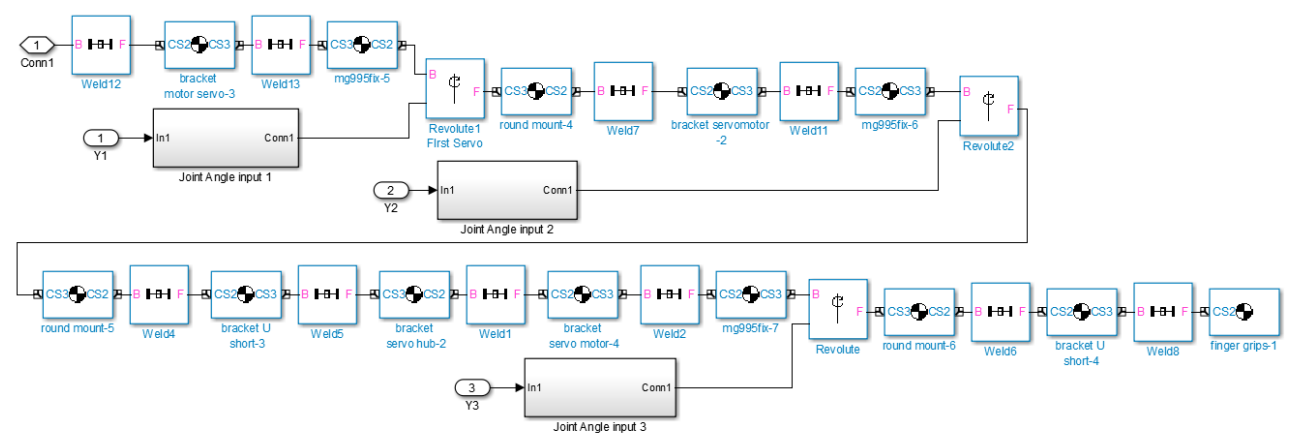

Fig. 5. SRF block diagram in SimMechanics.
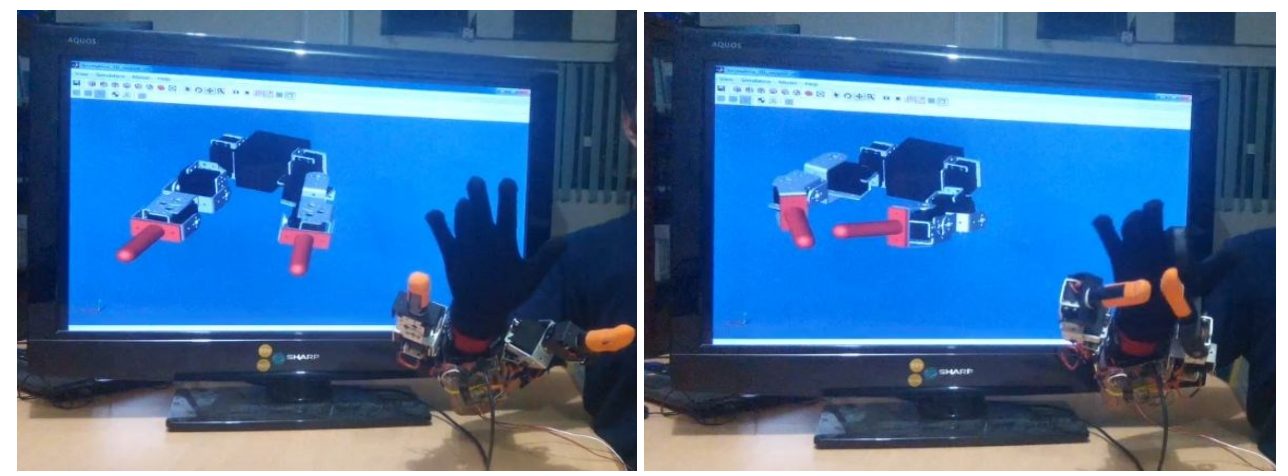

Fig. 6. SRF integrated with 3D Animation SimMechanics.

In this research, the SRF will be tested to perform object manipulation with ten-inch tablet. The test shows a promizing result in bimanual task. The SRF can hold a tablet while the human fingers operate the tablet.

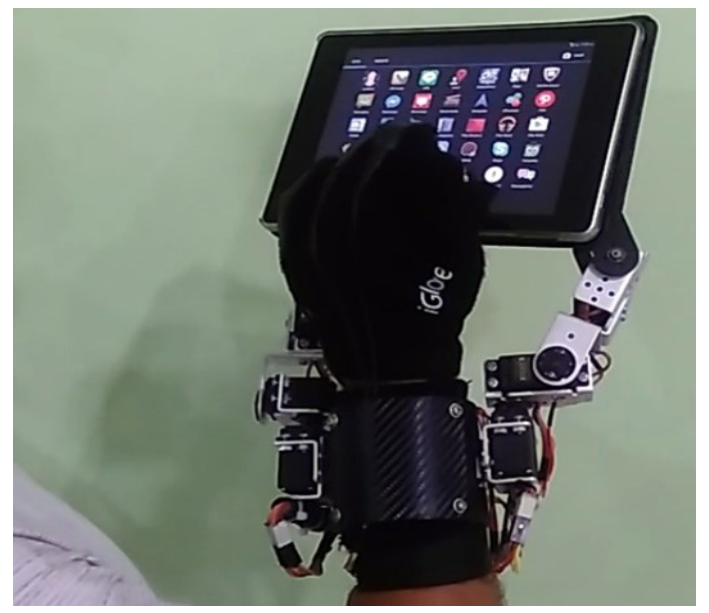

Fig. 7. Possible application of SRF for object manipulation. 


\section{Conclusion}

Forward kinematics can be used to study the trajectory of the end effector in both RT and $\mathrm{RF}$. Based on the experimental result, the robot and its 3D animation can be operated by a healthy person in real time. The robot also can do object manipulation with ten-inch tablet using single healthy hand. For future study, the robot will be explored for bimanual object manipulation using single human hand with the assistance of the SR fingers.

\section{References}

1. F. Y. Wu, H Asada, ASME Dynamic Systems and Control Conference, 1-8, (2014)

2. F. Y. Wu and H. H. Asada, 2015 IEEE International Conference on Robotics and Automation (ICRA), 6205-6212, (2015)

3. F. Y. Wu and H. H. Asada. IEEE Trans. Robot., 32,176-186 (2016)

4. F. Y. Wu, and H. Asada. in Robotics: Science and Systems X, University of California, Berkeley, USA (2014)

5. M. Ariyanto, R. Ismail, A. Nurmiranto, W. Caesarendra, Paryanto and J. Franke, 2016 IEEE EMBS Conference on Biomedical Engineering and Sciences (IECBES), Kuala Lumpur, 341-346,(2016)

6. R. Ismail, M. Ariyanto, W. Caesarendra, I. Haryanto, H. K. Dewoto and Paryanto, 2016 IEEE EMBS Conference on Biomedical Engineering and Sciences (IECBES), Kuala Lumpur, 335-340, (2016)

7. R. Ismail, M. Ariyanto, W. Caesarendra and A. Nurmiranto, 2016 International Seminar on Intelligent Technology and Its Applications (ISITIA), Lombok, 629-634, (2016) 